\title{
CHARGE CARRIER GENERATION CAUSED BY X-RAYS IN ORGANOMETALLIC MATERIALS*
}

\author{
J. GODLEWSKI ${ }^{a}$, R. Signerski ${ }^{a}$, G. JAROSz ${ }^{a}$, S. StizzA ${ }^{b}$ \\ AND M. BERRETTONI ${ }^{b}$ \\ ${ }^{a}$ Department of Physics of Electronic Phenomena, Technical University of Gdanisk \\ Narutowicza 11/12, 80-952 Gdańsk, Poland \\ ${ }^{b}$ Dipartimento di Matematica e Fisica, Universita di Camerino, 60123 Camerino, Italy
}

Organometallic compounds play an exceptional role among organic compounds. Due to their structure a number of these compounds demonstrate interesting properties in various phenomena. Organometallic compounds show special properties in the case of X-ray absorption owing to considerable differences between the absorption coefficients of metals and light elements. The analysis of processes of charge carrier generation as a result of X-ray absorption in chosen organometallic compounds is the subject of this paper. Spectral dependences of charge carrier photogeneration efficiency around the $\mathrm{Cu} K$-edge in copper phthalocyanine and copper acetylacetoniane have been specially considered. It has been observed that the photocurrent spectrum in these materials follows the absorption spectrum but mutual relation is not directly proportional. Experimental results connected with current-voltage and current-intensity characteristics have been analysed, as well. The obtained relationships have been compared with adequate results connected with charge carrier photogeneration in visible area and UV in organic materials. It has been observed that the X-ray photogeneration of charge carriers in the examined range of energy differs from the mechanisms of charge carrier generation in visible area and UV. The basic differences arise from the participation of secondary electrons, which are generated due to Auger electrons, Compton effect, and metal fluorescence. The process of charge carrier generation as a result of $\mathrm{X}$-rays absorption is characterized by strong recombination of charge carriers in channels in which charge carriers are generated by high energetic secondary electrons.

PACS numbers: $72.40 .+\mathrm{w}$

\section{Introduction}

A metal atom as a component of molecule of organometallic compound plays an exceptional role in the process of X-ray absorption. It results from a significant difference between the absorption coefficients of the metal and the remaining ele-

\footnotetext{
*Partly supported by the Committee for Scientific Research grant No. 2PO3B15409.
} 
ments of the compound [1]. In a spectrum of X-rays absorption we can distinguish the ranges where $\mathrm{X}$-ray absorption is determined by the metal atom from the ranges where absorption is connected with the remaining atoms of the molecule.

Special attention of this paper is paid to generation of charge pairs induced by $\mathrm{X}$-ray absorption by metal atoms. Generation of charge carriers in organometallic compounds plays an exceptional role in processes of physical chemistry, biology, and medicine as well as in potential application of these compounds. Moreover, this paper comprehends the analysis of spectral, voltage, and intensity dependences of photocurrent and an analysis of efficiency of charge carrier photogeneration. Two different organometallic compounds are used to perform the investigation: copper phthalocyanine $(\mathrm{CuPc})$ and copper acetylacetonate $\left(\mathrm{Cu}(\mathrm{acac})_{2}\right)$. Both of them contain copper atom and light elements. The chemical structures of copper phthalocyanine and copper acetylacetonate are presented in Fig. 1.

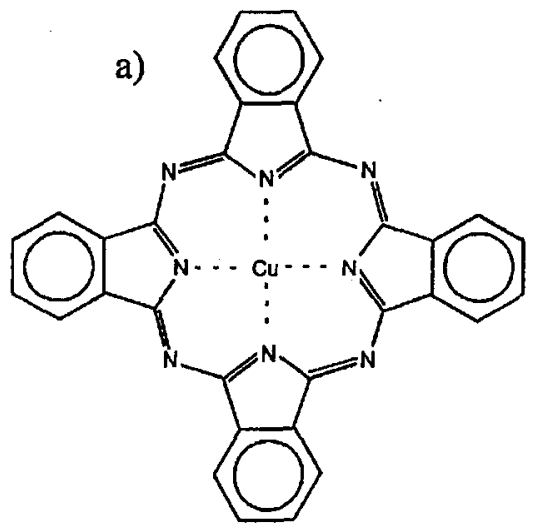

b)<smiles></smiles>

Fig. 1. The chemical structure of copper phthalocyanine ( $\mathrm{CuPc})(\mathrm{a})$ and copper acetylacetonate $\left(\mathrm{Cu}(\mathrm{acac})_{2}\right)(\mathrm{b})$.

The paper sums up a certain group of experimental results, among which the most important concerns the spectral dependence of efficiency of charge carrier generation $[2,3]$.

Referring to the known mechanism of charge carrier generation in the visible and ultraviolet ranges we discuss experimental results concerning charge carrier photogeneration by $\mathrm{X}$ radiation nearby the $K$-edge of copper. In this paper we put emphasis on differences and similarities between charge carrier photogeneration in the ultraviolet and X-ray ranges of electromagnetic waves.

\section{Charge carrier generation in the visible and ultraviolet ranges}

Absorption of electromagnetic radiation by organic materials in the visible and ultraviolet ranges can lead to charge carrier generation. Efficiency of this process is estimated as $10^{-4}$, when the external electric field does not exceed $10^{4} \mathrm{~V} / \mathrm{cm}$ [4]. Such low efficiency of generation results from the strong geminate recombination because the initial distance of electron and hole separation $r_{0}$ is much smaller than the Coulombic capture radius $r_{\mathrm{c}}$ defined by the equality between 
electrostatic and thermal energy

$$
r_{\mathrm{c}}=\frac{e^{2}}{4 \pi \varepsilon \varepsilon_{0} k T}
$$

where $\varepsilon_{0}$ and $\varepsilon$ are the permittivities of vacuum and the dielectric used, $k$ is the Boltzmann constant, $T$ is the absolute temperature, $e$ is the electronic charge.

The typical value of $r_{\mathrm{c}}$ amounts to $160 \AA\left(\varepsilon \varepsilon_{0}=3 \times 10^{-11} \mathrm{~F} / \mathrm{m}, T=300 \mathrm{~K}\right)$. The efficiency of charge carrier separation and escape depends on initial separation distance $r_{0}$ as well as on external electric field.

The process of charge carrier separation is described by Onsager theory [5], which predicts the linear dependence of separation efficiency on electric field strength for relatively low values of electric field

$$
f(E) \cong f(0)\left(1+\frac{1}{2} \frac{e}{k T} r_{\mathrm{c}} E\right),
$$

where $f(0)$ is efficiency of charge carrier separation for electric field equal to zero and it depends on the initial distance of charge carriers $r_{0}$.

The ratio of slope $(S)$ to intercept $\left(I_{c}\right)$ of this straight line to its abscissa amounts to

$$
\frac{S}{I_{\mathrm{c}}}=\frac{e^{3}}{8 \pi \varepsilon \varepsilon(k T)^{2}}
$$

Above relations were many times confirmed experimentally [4].

If intensity of light is not too high and mechanism of photogeneration is described by Onsager model, the dependence of photogeneration efficiency on light intensity is also linear. Moreover, if radiation is completely absorbed by sample the dependence of photogeneration efficiency on wavelength does not appear (for $r_{0}=$ const).

It must be noticed that initial distribution of charge carrier is not always well described by a $\delta$-function (it means $r_{0}=$ const.). In some cases this distribution is of a different form [4].

\section{Charge carrier generation by $\mathrm{X}$-ray radiation}

In this chapter we discuss experimental investigation of photoconductivity of copper phthalocyanine and copper acetylacetonate in the range of X-rays. The chemical structure of investigated compounds is shown in Fig. 1.

Figure 2 presents calculated dependence of linear absorption coefficient on $\mathrm{X}$ radiation energy. This coefficient defined by the following relation:

$$
\varepsilon=\frac{1}{d} \ln \frac{I_{0}}{I}
$$

(where $d$ is the sample thickness, $I_{0}$ is the intensity of incident radiation, and $I$ is the intensity of radiation going out) was calculated according to the following relation:

$$
\varepsilon=\left[\left(\frac{\mu}{\rho}\right)_{\mathrm{Cu}} \frac{\mu_{\mathrm{Cu}}}{N_{\mathrm{A}}}+\sum_{i}\left(\frac{\mu}{\rho}\right)_{i} \frac{\mu_{i}}{N_{\mathrm{A}}}\right]
$$

by the use of the known values of atom absorption coefficient $(\mu / \rho)[1]$. 


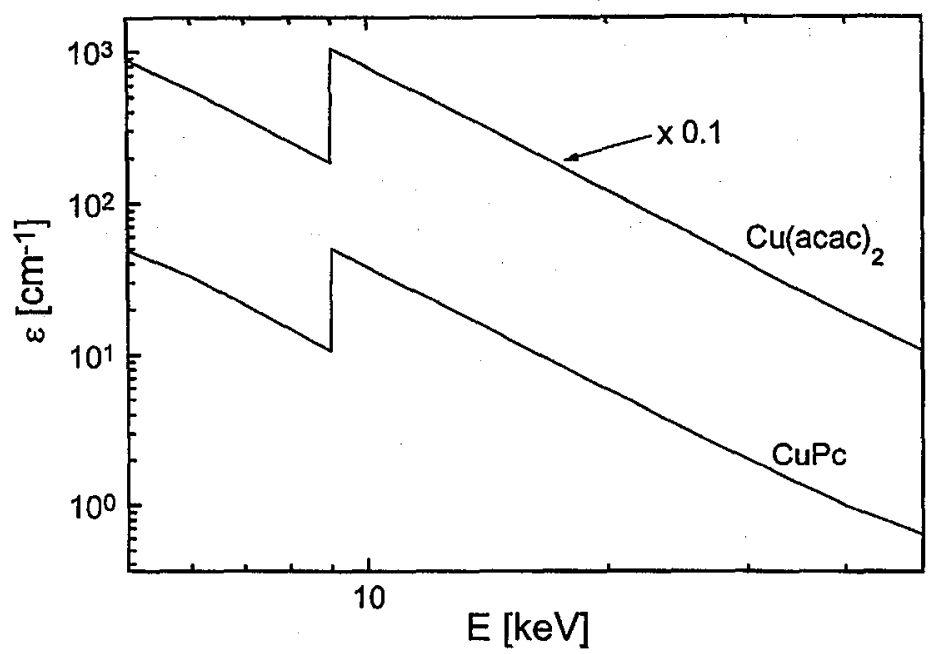

Fig. 2. Calculated values of linear absorption coefficient of X-rays as a function of energy for $\mathrm{CuPc}$ and $\mathrm{Cu}(\mathrm{acac})_{2}$.

In relation (5) $\mu_{\mathrm{Cu}}$ and $\mu_{i}$ mean atomic weight of copper and light elements, and $N_{\mathrm{A}}$ is Avogadro's constant. The apparent stroke increase in coefficient $\varepsilon$ is connected with the copper $K$-edge of absorption. Photocurrent spectra of CuPc and $\mathrm{Cu}(\mathrm{acac})_{2}$ nearby the $K$-edge of copper $(8.98 \mathrm{keV})$ are presented in Figs. 3 and 4. Experimental investigations were carried out on pills, which thicknesses assure a complete absorption of $\mathrm{X}$ radiation.

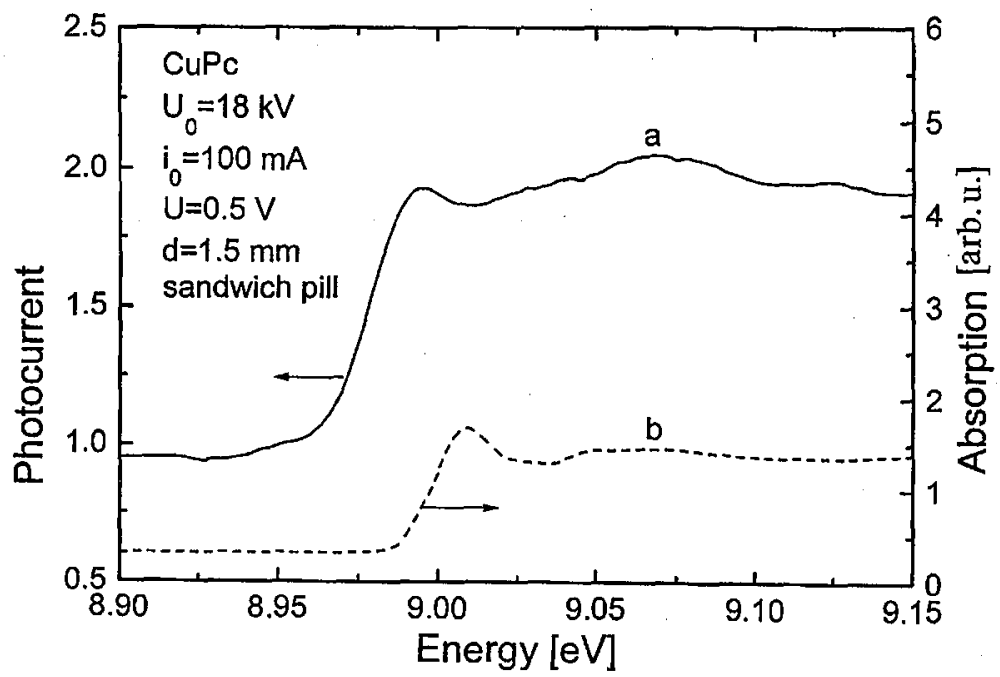

Fig. 3. Spectral dependence of $i_{+}$photocurrent $a$ and of absorption $b$ for $\mathrm{CuPc}$ in the sandwich system. 


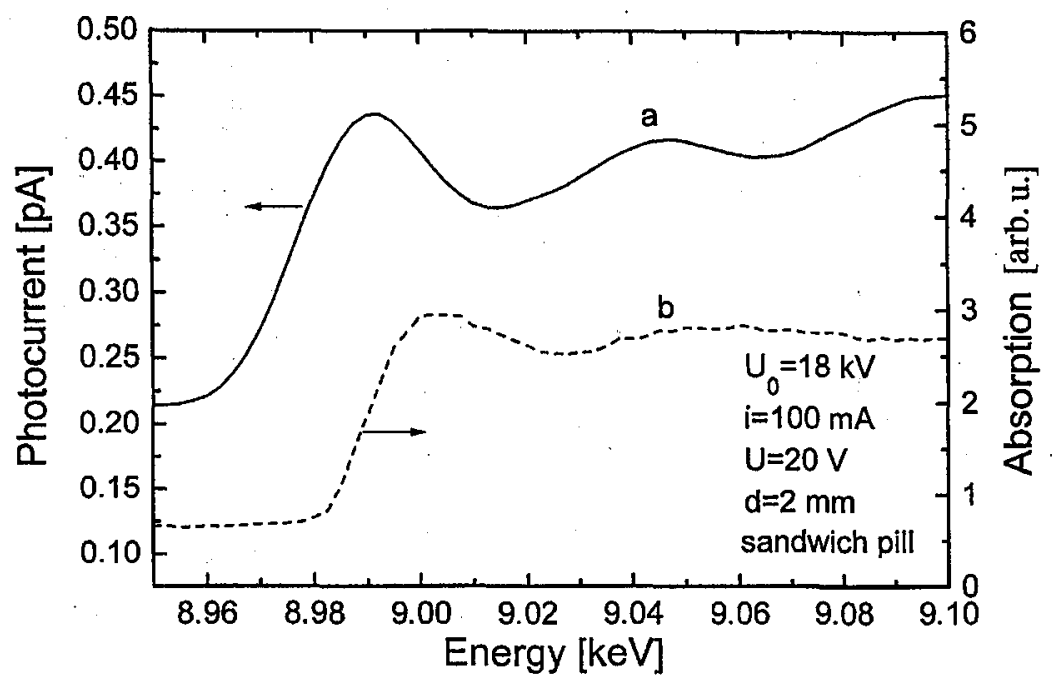

Fig. 4. Spectral dependence of $i_{+}$photocurrent $a$ and of absorption $b$ for $\mathrm{Cu}(\mathrm{acac})_{2}$.

The measurement apparatus (EXAFS-Rigaku system) enabled us to observe a fine structure of absorption and photocurrent spectra.

The value of linear absorption coefficient below the absorption edge amounts to $10 \mathrm{~cm}^{-1}$, and for a higher energy than the edge amounts to $50 \mathrm{~cm}^{-1}$ (see Fig. 2). It means that samples, which are 1.5 or $2 \mathrm{~mm}$ thick, can almost completely absorb the incident radiation ( $80 \%$ and more).

The observed twice increase in photocurrent for the energy higher than the $K$-edge of copper does not result from the increase in a number of absorbed photons. This increase confirms that the efficiency of charge carrier generation connected with X-ray absorption by a copper atom is higher than the efficiency of generation induced by remaining atoms of molecule.

The increase in efficiency of charge carrier generation over the $K$-edge of copper can be caused by appearance of Auger electrons and photons of characteristic fluorescence of the copper atom.

Figures 5 and 6 present experimental current-voltage and current-intensity relations for the vacuum evaporated sample illuminated by a full spectrum of X-ray tube. The apparent changeable slope of characteristics presented in Fig. 5 as well as deficiency of proportion of current to radiation intensity (Fig. 6) indicate that these dependences are not in agreement with Onsager model (Eq. (2)). The reason for it can be that the initial separation distance of charge carriers generated by $\mathrm{X}$ radiation is higher than the Coulombic capture radius. Similar relations were also obtained for different organic materials $[6,7]$.

\section{Conclusions}

The experimental results presented above allow us to conclude that the mechanism of charge carrier photogeneration is not determined by the geminate recombination but rather by the bimolecular recombination. It can cause a surprise 


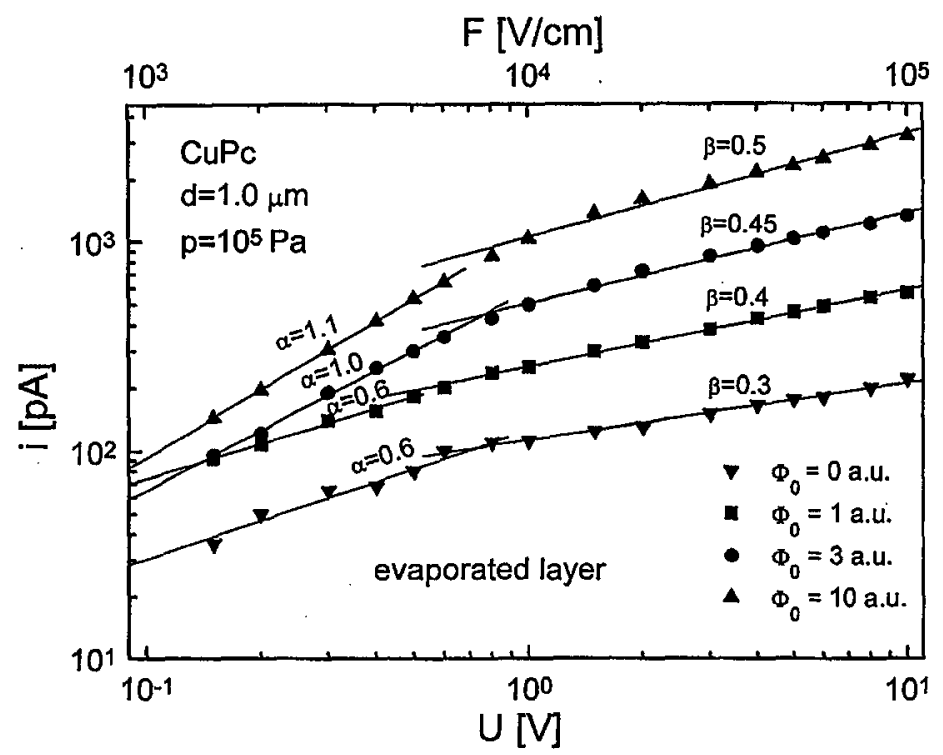

Fig. 5. Current-voltage characteristics for various $\mathrm{X}$-ray intensities at atmospheric pressure. Parameters $\alpha$ and $\beta$ denote slopes of the characteristics.

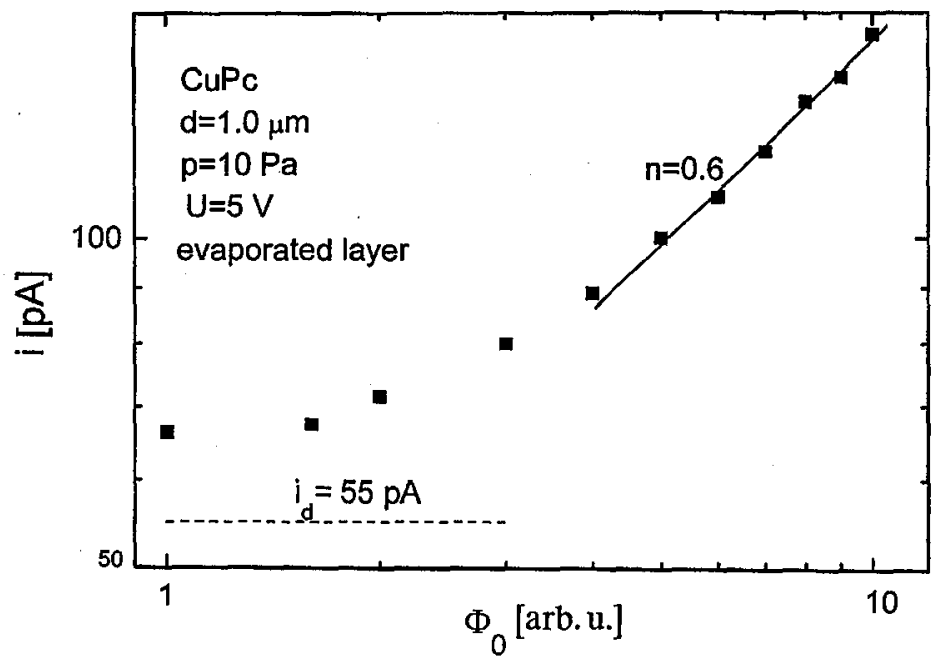

Fig. 6. Photocurrent - light intensity characteristics at low pressure.

because the results presented in Figs. 5 and 6 concern thin samples, in which the average transit time of charge carrier for relatively high electric field is short and the probability of avoidance of recombination is high. However, it must be noticed that in the case of illumination of molecular materials by $\mathrm{X}$ radiation the charge carriers are mainly generated by Compton and secondary electrons in small blobs in which local density of charge is high $[4,8]$. It creates convenient conditions for appearance of bimolecular recombination. 
Finally, in order to explain the increase in charge carrier generation connected with $K$-edge absorption of copper we should take into consideration also Auger electrons as well as the characteristic fluorescence of the metal. Primary photoelectrons generated directly by photons in photoelectric process possessing almost $9 \mathrm{keV}$ energy have too little kinetic energy to creation of charge pair, because the inscrutable energy is about $30 \mathrm{eV}[4,8]$.

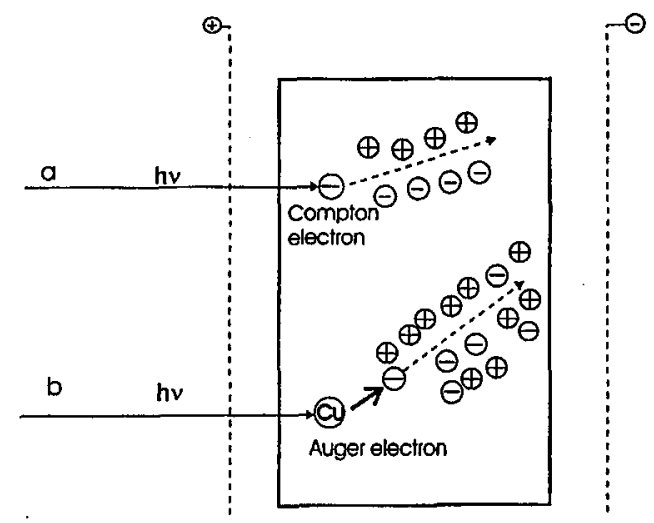

Fig. 7. Possible mechanisms of charge carrier generation by X-rays in organometallic materials, (a) Compton electrons, (b) Auger electrons.

The mechanisms of charge carrier generation pointed above are diagrammatically presented in Fig. 7. They can be a base of a qualitative interpretation of the obtained experimental results.

\section{References}

[1] Handbook of Spectroscopy, Ed. J.W. Robinson, CNR Press, Rome 1974.

[2] S. Stizza, M. Berrettoni, R. Signerski, J. Godlewski, Chem. Phys. Lett. 253, 113 (1996).

[3] M. Kozłowski, J. Godlewski, H. Sodolski, M. Berrettoni, S. Stizza, in: Proc. Fourth Intern. Conf. on Intermolecular Interaction in Matter, Eds. K. Sangwal, J.M. Olchowik, E. Piewla, Technical University of Lublin, Lublin 1997, p. 109.

[4] M. Pope, C.E. Swenberg, Electronic Processes in Organic Crystals, Clarendon Press, New York 1982.

[5] L. Onsager, Phys. Rev. 54, 554 (1938).

[6] R.G. Kepler, F.M. Coppage, Phys. Rev. 151, 610 (1966).

[7] J. Godlewski, R. Signerski, J. Kalinowski, S. Stizza, M. Berrettoni, Mol. Cryst. Liq. Cryst. 252, 145 (1994).

[8] G. Klein, Mol. Cryst. Liq. Cryst. 47, 39 (1978). 